\title{
EL DERECHO DE LA SALUD EN LA GESTIÓN PÚBLICA. LA EXPERIENCIA DEL ÁREA DE BIOÉTICA DEL MINISTERIO DE SALUD DE LA PROVINCIA DE CÓRDOBA (ARGENTINA) EN ALGUNAS APLICACIONES DE ESTE DERECHO
}

\author{
THE RIGHT TO HEALTH IN PUBLIC MANAGEMENT. \\ THE EXPERIENCE OF THE BIOETHICS AREA OF THE \\ MINISTRY OF HEALTH OF THE PROVINCE OF CORDOBA \\ (ARGENTINA) IN SOME APPLICATIONS OF THIS RIGHT
}

\section{Andrés F. Toledo ${ }^{1}$}

DOI: https://doi.org/10.37767/2591-3476(2020)01

RESUMEN:

Esta ponencia pretende fundamentar y contextualizar, desde un enfoque de derechos humanos y desde la misma disciplina Bioética; lo actuado por el Área de Bioética del Ministerio de Salud de Córdoba, Argentina en el año 2019. Se parte del análisis de dos iniciativas que se están plasmando en los hospitales públicos de la provincia como son: la cartelería inicial, el posterior ciclo de reuniones formativas sobre "derechos y responsabilidades de los pacientes", y la implementación del "consentimiento informado general de internación en hospitales públicos". Al final, se plantean los desafíos pendientes para que la asistencia pública en esta materia sea pionera, primera garante y promotora de los derechos de los pacientes que asiste.

\section{ABSTRACT}

This paper aims to provide a basis and context for the actions taken by the Bioethics Area of the Ministry of Health of Córdoba, Argentina, in 2019, from a human rights perspective and from the discipline of Bioethics itself. It is based on the analysis of two initiatives that are being implemented in the public hospitals of the province: the initial poster, the subsequent cycle of training meetings on "patients' rights and responsibilities", and the implementation of "general informed consent for hospitalization in public hospitals". In the end, the pending challenges are posed so that public assistance in this area can be a pioneer, the first guarantor and promoter of the rights of the patients it assists.

\footnotetext{
1 Doctor en Filosofía (UCA). Magíster en salud pública (UNC) y en ética biomédica (UCA), Miembro del equipo permanente Área de Bioética del Ministerio de Salud de Córdoba, Argentina. Docente universitario de bioética del Instituto Universitario De Ciencias Biomédicas de Córdoba (IUCBC), Tutor Universitario de Universidad Blas Pascal. E-mail: andrestoledo14@hotmail.com. ORCID iD: https://orcid.org/0000-0002-1175-8568.
} 
PALABRAS CLAVE: Salud pública; Bioética, Derechos y responsabilidades de los pacientes; Consentimiento informado general de internación; Área de bioética de Córdoba; Argentina.

KEY WORDS: Public health; Bioethics; Patients' rights and responsibilities; General informed consent for hospitalization, Bioethics area of Córdoba; Argentina.

\section{Introducción y problemática}

La asistencia sanitaria es un derecho humano de segunda generación, postulado por la Organización de Naciones Unidas (ONU), en la Declaración Universal de Derechos Humanos de $1948^{2}$, que consiste en la provisión de ayuda médica o sanitaria, a personas que se enferman, o sufren algún daño corporal, quienes deben contar con la debida protección para que su salud sea restablecida.

Por eso, los derechos de segunda generación se dice que son materiales, ya que permiten que el resto de los derechos humanos puedan efectivizarse y realizarse de "manera concreta y real'3, buscando que existan igualdades y similares oportunidades iniciales en todos los miembros de esa sociedad, más allá de su "posición original", tal como fue enunciada por Rawls ${ }^{4}$ y otros pensadores de la teoría política contractual.

Así, no solamente se necesita un derecho a la vida (derecho de primera generación), sino que también - en la misma medida y tan necesario como el derecho a la vida- se necesita un derecho a la asistencia sanitaria. Ésta, como todo derecho humano, debe tener cierta universalidad, lo que implica que debe llegar a todos los ciudadanos de manera real, garantizándose el acceso para su concreción y ejercicio efectivo.

Por eso, desde este enfoque; el derecho humano a la asistencia sanitaria tiene enorme relación con la asistencia pública estatal, y cobra peculiar relevancia si se reflexiona desde el contexto provincial y nacional.

Por otra parte, la Bioética como disciplina, nace en los comienzos de los años 70, en los Estados Unidos. En el año 1973 ésta fue una de las pioneras en asumir y proponer los primeros derechos de los pacientes como así también, desde sus distintas teorías éticas, "siempre ha propuesto en primer lugar, el principio de autonomía con su aplicación, que es el consentimiento informado"s. Sin embargo, aunque parezca extraño, la misma disciplina, si bien tiene un desarrollo importante en estos temas (derechos de los pacientes y consentimiento informado), no ha tenido ni tiene una reflexión especial sobre estos a nivel práctico y, en particular desde la asistencia sanitaria en la gestión pública o estatal.

Por eso, no es llamativo que a nivel de implementación, no haya trabajos ni se registren experiencia donde se relacionen los derechos y responsabilidades de los enfermos en el ámbito público, ni muchos menos que refieran sobre cómo se plasman los consentimientos informados en los hospitales estatales.

\footnotetext{
2 Organización de Naciones Unidas (ONU) 1948. Declaración Universal de Derechos Humanos. Art 25.

3 CORTINA, Adela (1997), La ética de la sociedad civil. p. 105.

4 RAWLS, John (1979), Teoría de la justicia p.29.

5 GAFO, Javier (2003), Bioética Teológica. p.27.
} 
Así, este trabajo, por un lado, pretende reflexionar de manera inicial sobre el tema de derechos y responsabilidades de los pacientes a nivel público desde una mirada Bioética, para luego centrarse en dos iniciativas recientes llevadas a cabo por el Área de Bioética del Ministerio de Salud de la provincia de Cordoba en Argentina, que intenta visibilizar estos derechos.

Para concluir, se afirma, que el garante por excelencia de este derecho a la asistencia sanitaria, es sin duda, el Estado. Esta institución con sus leyes, decisiones políticas y presupuestos, ordena el ejercicio de este derecho para todos los ciudadanos, teniendo una mayor responsabilidad para con las instituciones de salud que el mismo Estado gestiona. De allí que será siempre un desafío, que el mismo Estado, que es el garante del ejercicio de este derecho, no sea el primero en vulnerarlos o no respetarlos.

\section{La Bioética en la gestión pública}

Como se acaba de afirmar, nuestra posición es, que no debería haber una reflexión especial, que vincule derechos y responsabilidades de los enfermos y la gestión sanitaria pública estatal. La literatura bioética es abundante, y ya se encuentra en una etapa de consolidación ${ }^{6}$, por eso, se sostiene que lo mejor es echar mano de estos contenidos, y tratar de hacer acciones de aplicación. En otras palabras, si bien no podemos soslayar que existen muy buenos trabajos que vinculan salud pública y bioética ${ }^{7}$, todos estos trabajos y reflexiones abordan la temática desde un contexto general abstracto, y no lo refieren a un marco de salud público concreto, y con las características tan propias, que tiene la asistencia sanitaria estatal. Por eso, estos trabajos contienen elementos válidos para la reflexión específica, pero por otro lado, no terminan de dar cuenta de la realidad concreta con qué se vive la salud en los ámbitos públicos provinciales.

¿A qué nos referimos? Según el Barómetro de la Deuda Social Argentina de la UCA, en un trabajo que abarca los años 2010 al 2016, analiza el acceso a la salud en población urbana argentina desde una mirada multidimensional de la pobreza. En este informe afirma "que 3 de cada 10 argentinos se atiende en la asistencia pública"s, y que si tomamos estos datos en el 2016, este porcentaje va en franco aumento. Asimismo, según sus resultados, el $70 \%$ de los pobres y el $80 \%$ de los indigentes, son atendidos por la asistencia pública. Asimismo indica que la población que no está dentro de la línea de pobreza ni de indigencia, son cada vez más los que concurren a los hospitales públicos para su atención. Y los que tienen vulnerados uno de sus derechos, concurren cerca del $50 \%$, y en los que tienen más de dos derechos vulnerados es superior al 50 \%, aumentado su número si la medición se realiza cerca del 2016.

Esto ya brinda un cuadro de situación cuando hablamos de asistencia pública. Si bien no hace falta un estudio investigativo especial para identificar que la salud publica atiende a la población más vulnerable económicamente, socialmente y sanitaria, es importante poner un número a lo que se vive cada día en los pasillos y consultorios de los centros públicos de atención en salud. Siguiendo el mismo trabajo, dice que solo 2 de cada 10 personas con vulneración de derechos va a consultas de salud mental, aumentando la proporción en poblaciones más acomodadas, y que 3 de cada 4 pobres e indigentes no tuvieron atención odontológica.

6 Cfr. GRACIA, Diego (2004), Como Arquero al Blanco, p.83.

7 TEALDI, Juan Carlos (2007) Retos para la bioética en el camino de la salud púbica en América Latina p.229.

8 Barómetro de la Deuda social Argentina. (2016) La salud en población urbana argentina desde una mirada multidimensional de la pobreza. p.12. 
Así, cuantificando estos datos visibles, se puede concluir que, al hablar de derechos y responsabilidades de los enfermos y de consentimiento informado, se debe tomar en cuenta estos datos, ya que modifican de manera determinante, cómo se deben aplicar y entender las reflexiones generales o teóricas de estos temas.

Con esto nos referimos a que la contextualización en la salud pública de estos derechos, hacen de los mismos más que un axioma a defender o postulado a proclamar; se transforman en una meta a alcanzar, y en un objetivo político y práctico de trabajo.

Así, desde estas constataciones anteriores, como eje de este trabajo se quiere presentar una bioética que asume las reflexiones teóricas como algo del patrimonio constitutivo de la disciplina, pero que por tratarse de salud pública, lo que le interesaría de sobremanera ver, son las formas concretas de aplicación y cómo se podría hacer para que estos conceptos "bajen" de manera concreta en los pacientes que se atienden la asistencia pública, en especial aquellos con alta vulnerabilidad de sus derechos sociales y económicos.

Por eso, nos parece que, al hablar de bioética y la asistencia púbica, no hay que inventar una nueva bioética, o una nueva declaración de derechos de los pacientes, o nuevos requisitos de implementación del consentimiento informado, sino que necesitamos impactar con decisiones gubernamentales, con programas detallados de implementación, y con presupuestos acordes a los programas operativos diseñados, para que estos dere- chos tengan realidad y visibilidad concreta en la población alcanzada por la cobertura estatal.

En esta dirección nos parece significativo destacar las palabras de un gran bioeticista latinoamericano chileno, el Dr. Miguel Kottow quien advertía sobre la necesidad de que la Bioética y especialmente la latinoamericana sea una "bioética viva", es decir operativa y llena de acciones concretas para el bien de la comunidad y de los pacientes ${ }^{9}$.

Esta bioética "viva" es lo que intenta el área de bioética, practicar y desarrollar cada día en su ser y hacer.

\section{El Área de Bioética y sus acciones en la concreción de derechos de los pacientes}

El "Área de Bioética", nace en 1998 y en la actualidad depende de la Secretaria de Prevención y Promoción de Salud, del Ministerio de Salud de la Provincia de Córdoba. A lo largo de estos años, ha tenido distintas modificaciones y competencias en el campo disciplinar de la bioética. En la actualidad, los Objetivos y Funciones del Área de Bioética han sido definidos por la Resolución Ministerial № 1264, con su anexo 1, de fecha 23 de noviembre de 2017 donde figura la promoción y cuidado de los derechos de los pacientes. ${ }^{10}$

En este contexto, es que, en el año 2019, se han implementado dos acciones que buscan expresar mejor cómo la bioética en instituciones púbicas tiene relación directa a un hacer. Ellos son: (1) Los derechos y responsabilidades de los pacientes y (2) el formulario de consentimiento informado general de internación. Ambos documentos se consignan en la sección anexo de este escrito.

Veamos más en detalle estas dos acciones:

\footnotetext{
9 memorias semana provincial de la bioética 2019- disponible en https://www.cba.gov.ar/area-de-bioetica/semana-provincial-de-la-bioetica/jornada-decierre-viernes-16-de-agosto/.

$10 \mathrm{cfr}$. https://www.cba.gov.ar/area-de-bioetica/objetivos-y-funciones/.
} 


\section{Los derechos y responsabilidades de los pacientes en los hospitales públicos}

Una premisa fundamental para abordar este tema fue presentar los derechos y las responsabilidades de los pacientes, como binomios que se deben entender juntos y en mutua correlación. Por eso, como afirma David Ross y otros, que "no hay derechos sin deberes $^{\prime \prime 1}$, ni hay deberes que no impliquen algún tipo de derecho, por una cuestión de presentación léxica y gráfica para esta iniciativa, se adoptó el término "responsabilidades" en vez de usar la palabra deberes.

Esta propuesta nace de una demanda que se está dando en algunos hospitales públicos, donde muchas veces se generan tensiones en la relación médico y paciente, terminando en algunas ocasiones en situaciones de violencia, ya sea verbal o gestual. Así fue como varios comités de bioética institucional propusieron la necesidad de general cartelería sobre estos derechos de los pacientes con sus correspondientes responsabilidades.

También en este tema, se muestra una clara diferencia entre la asistencia sanitaria de gestión privada y la pública, ya que no hay clínica, centro o instituto de salud privado que no cuente con su cartelería correspondiente, siendo por el contrario difíci encontrar estos mismos derechos expuestos en los hospitales públicos.

Observando esta gran carencia de una buena cartelería que planteara los derechos de los pacientes, desde el Área de Bioética se diseñó y solicitó que se proveyera una buena cantidad de banners y folleterías, para que llegara de manera masiva a todas las dependencias del Ministerio de Salud de la Provincia.

Así, con un grupo de estudio creado ad hoc, con algunos miembros de los distintos comités hospitalarios de la provincia, es que se confeccionaron una serie de derechos y responsabilidades de los pacientes, especialmente pensados para aquellos pacientes que generalmente se atienden en los hospitales públicos.

Al mismo tiempo que se hizo esta cartelería, se han llevado a cabo una serie de charlas en distintos hospitales con esta temática, como también se ha brindado una propuesta de formación a través de cursos gratuitos a distancia, auto asistidos, que se encuentran disponibles en la página web del área de bioética ${ }^{12}$. Todas estas iniciativas buscan que estos derechos no sean una imposición, sino una convicción en el efector de salud, quien debe respetar esos derechos sin entenderlos como una carga más, sino una manera de hacer mejor medicina.

A modo de ejemplo, se adjunta folletería donde se observan los derechos trabajados. Si bien no se trata de nuevos derechos, son conceptualizados de una manera sencilla y adaptada, para la mejor compresión de los todos los pacientes.

Así, los derechos expresados son: Derecho a recibir atención sanitaria adecuada, gratuita y sin discriminación, a recibir un trato digno y respetuoso cuidando su intimidad, a que sus datos personales e historia clínica sean confidenciales, a recibir información en un lenguaje claro y comprensible para tomar su propia decisión y luego a firmar su consen-

11 ROSS, David. (1994). Lo correcto y lo Bueno. p. 63. 
timiento informado. Asimismo se plantea la posibilidad de aceptar o rechazar cualquier tratamiento procedimiento que se le ofrezca, contar en la compañía de algún familiar, tener visita y apoyo espiritual o religioso, como así también a realizar una consulta de carácter bioético al comité hospitalario de bioética.

Así, por ejemplo, se sabe que desde el aspecto material y de funcionamiento de los centros de salud, muchas veces los presupuestos ni el personal de mantenimiento no alcanzan por los destrozos, desidia y la manera como se manejan los pacientes y personal en el uso de la cosa pública. En esto, también la bioética tiene algo que decir, porque lo público es cosa de todos y por tanto el cuidado de lo material es tarea tanto de los agentes de salud y usuarios del sistema de salud pública.

Entre las responsabilidades ${ }^{13}$, se señala de tratar con respeto y sin violencia el personal de salud que lo atiende, cuidar la propia salud siguiendo las recomendaciones de profesionales de salud que te trate, informarse, preguntar y leer atentamente lo que se tiene antes de firmar el consentimiento informado, proporcionar al equipo de salud toda la información requerida, necesaria y cierta, sobre la identidad del paciente y su condición de salud. Respetar, el paciente y sus familiares, las normas y reglamentos del establecimiento en el que se atiende. Evitar conductas que molesten a otras personas. Cuidar y hacer correcto uso de las instalaciones y equipamiento del establecimiento de salud

De vuelta, lo del punto anterior, no es que se pongan nuevas deberes o responsabilidades, sino que se ha tomado la teoría general sobre derechos y obligaciones de los pacientes, intentando simplificar, en un lenguaje más sencillo y cercano estas responsabilidades, para que sea entendido por los pacientes, seleccionando aquellos que tienen que ver más con la realidad pública sanitaria.

\section{Consentimiento informado para internación general en los hospitales públicos}

Recordemos que el consentimiento informado nace con la bioética y es uno de los institutos mejor consolidado de la misma ${ }^{14}$. De hecho, el consentimiento trae aparejado un quiebre en el paradigma secular de la relación médico-paciente, que se conoce como el paternalismo médico.

Con el consentimiento informado se reconoce que toda persona debe ser preguntada e informada y que puede decidir de manera afirmativa o negativa, sobre cualquier práctica o procedimiento médico que se le proponga sobre su propio cuerpo.

De hecho, más allá del requerimiento bioético que tiene esta práctica, es una obligación legal solicitarlo y hay un sinnúmero de normativas que lo estipulan y establecen, como por ejemplo nuestro nuevo Código Civil y Comercial que en sus art.58 y 59 que lo expresan claramente.

Cabe aclarar que a pesar de estar establecido legalmente y bioéticamente, en los hospitales públicos de la provincia, solo existía el consentimiento informado para tratamientos específicos, como cirugías o alguna práctica invasiva, donde el riesgo del paciente es alto.

13 De vuelta se aclara que se decidió poner "responsabilidades" en vez de deberes porque nos pareció que iba a favorecer una mejor comprensión y llegada a los pacientes. Concretamente, algunos miembros de esta comisión de trabajo, opinaron que la palabra deberes, generaba imposición y obligación dura, siendo contrario al espíritu de los objetivos de esta propuesta que son llegar a los pacientes, desde los aspectos educativos y formativos. Lo ideal de toda responsabilidad es no usar lo coercitivo sancionatorio, sino la colaboración voluntaria de los involucrados.

14 SIMON LORDA, Pablo (2000), El consentimiento informado. p 21. 
En contraste resultaba que un ciudadano que se internaba por cualquier patología, aunque no revista gravedad, empezaba su internación en los hospitales públicos, sin que quede ningún registro donde conste lo que se explicó qué le iban a hacer, o que le podría pasar en esa internación, o cuáles eran sus derechos, como tampoco como sus responsabilidades como paciente.

Por eso, se vio la necesidad de proveer un consentimiento informado general de internación, sin reemplazar a los específicos que saben tener cada servicio médico. Es decir, se ofrece un consentimiento informado que apunta a la internación hospitalaria con todo lo que ello implica cuando alguien se interna y vive todas esas horas en la institución de salud, aunque sean procedimientos médicos de rutina o tratamientos con pocos eventos adversos. Esto parte del supuesto que el respeto del derecho de los enfermos implica que exista un consentimiento informado para esta internación hospitalaria.

En el formulario de consentimiento informado general de internación, se le informa que la enfermedad que padece puede tener un curso cambiante de tratamiento y se le dice, que, en todo momento de su estadía internado, se le brindará la mejor atención posible, que también se guardará su intimidad y confidencialidad, que también podría haber -bajo supervisión experta- la visita de personal médico en formación y que puede recibir asistencia religiosa o de algún otro tipo según sus deseos. También se le informa que puede consultar a un comité de bioética y también que, en caso de gravedad, puede expresar sus directivas de voluntades anticipadas y con la opción de aceptar o rechazar tratamientos que sean fútiles a nivel terapéutico y solo generan sufrimiento al paciente.

Conectando con la otra iniciativa, nos ha parecido que incorporar un consentimiento informado general de internación, es un paso muy importante para que se expliciten estos derechos de los pacientes, aunque siempre quedan como desafíos, que este valioso instrumento que expresa derechos y manifiesta las libertades fundamentales de toda persona no sea un papel más de la burocracia administrativa, la cual lamentablemente, existe en el Estado. Por otra parte, también se encuentra el desafío de que con el paso del tiempo no haya un relajamiento o una falta de recursos materiales, tan mínimos como dos hojas fotocopiadas, que haga que deje de aplicarse o brindarse el consentimiento informado en los hospitales públicos, y por ende, por estas coyunturas y otras, destruyan o fagociten tan importantes y centrales iniciativas.

A la vez que se ha terminado este consentimiento general de internación, ya se está trabajando y proyectando confeccionar unos consentimientos específicos para internación en unidades de cuidados críticos para adultos y pediátrica que están faltando, y son solicitados por las instituciones públicas de la provincia.

Ambas iniciativas, derechos y responsabilidades del paciente, como el consentimiento informado general de internación, se han presentado a las autoridades ministeriales de salud, para que pueda implementarse en las estructuras hospitalarias junto con lo indicativo que puede realizar el área de bioética. El objetivo es que sean acompañados por una resolución ministerial para que tenga mejor recepción y aceptación en el personal de salud en sus distintos niveles.

\section{Los desafíos de la bioética en el cuidado de los derechos de los pacientes, en la gestión pública.}


Para terminar, se quiere volver, al informe del Barómetro de la Deuda Social Argentina, antes citado, porque, si por un lado daba cuenta de que la mayoría de la población vulnerable son asistidos por los hospitales públicos, también se nos informa que son los más pobres e indigentes (vulnerados ${ }^{15}$ ), los que más sufren el uso de la asistencia sanitaria estatal. Son ellos los que tienen las horas de espera más larga (de 3 a 4 hs.) para que los atiendan, y hasta 3 a 4 meses para conseguir un turno para algún estudio complementario o regresar para que lo vuelva ver su médico tratante ${ }^{16}$.

La misma bioética, sostiene, que la atención pública, bajo ningún punto de vista, puede ser sinónimo de baja calidad o de menor eficiencia. De hecho, a nivel médico tecnológico, hoy por hoy, hay hospitales de la Provincia de Córdoba que cuentan con los mismos recursos humanos y técnicos, que los mejores centros privados de salud de tercer nivel de atención, y esto está muy bien, pero cuando se trata de la atención cotidiana y habitual, no se puede decir lo mismo, ya que son constatables las enormes falencias que existen.

Por eso, la atención sanitaria estatal, debe mejorar sus procedimientos y organización y revisar sus prioridades sanitarias. Esto no solamente se observa a nivel de infraestructura, de administración; sino que también debe abarcar, las dimensiones educativas, epidemiológicas y de las enfermedades prevalentes endémicas, que no son pocas en nuestra región.

El desafío para la bioética cuando tiene enclave estatal es que tiene que ser una bioética con sentido crítico, y en cierta medida con opinión independiente con respecto a todo organismo oficial ministerial.

Ese elemento "crítico" de la bioética, no necesariamente debe ser molesto o cuestionador de la gestión estatal, sino que se trata más bien, de aportes para que la salud pública mejore cada día más. La bioética, sin duda tiene un significativo potencial de generar capacitación y conciencia; y su transversalidad interdisciplinaria, puede hacer un aporte significativo, para que la atención sanitaria estatal, sea de vanguardia en sus propuestas y con eficientes resoluciones a los problemas sanitarios más acuciantes.

Si esto es verdad, también hay que decir, que estructuras como las que tiene el Ministerio de Salud de Córdoba con su Área de Bioética, implican que no sea una área de tipo "decorativa" y casi olvidada en la gestión concreta, sino que es de desear que sea tenida cada vez más en cuenta en las prácticas cotidianas médicas. Su relevancia justamente reside porque el Área de Bioética, no tiene como objetivos situaciones críticas o urgentes, sino que sus objetivos hacen referencia a lo importante e imprescindible que se debe dar en la relación sanitaria.

Por eso, desde el Área de Bioética se sostiene que la bioética es una herramienta para promover el debate público en temas éticos sobre la vida y la ciencia ${ }^{17}$ y como toda herramienta, su sentido está, en su uso constante y permanente.

Señalamos como último desafío, se tiene que hacer una bioética, no solo pública o aten-

15 Cfr. Ponencia del Dr. Miguel Kottow. Conferencia Inaugural Semana Provincial de la Bioética 2019 "vulnerabilidad, un concepto controversial". Disponibles: https://www.cba.gov.ar/area-de-bioetica/semana-provincial-de-la-bioetica/jornada-de-cierre-viernes-16-de-agosto/.

16 Cfr. Barómetro de la deuda social Argentina. (2016) La salud en población urbana argentina desde una mirada multidimensional de la pobreza. p.12.

17 Cfr. Organización de las Naciones Unidas para la educación, la ciencia y la cultura. (UNESCO). 2016. Manual de Bioética para Periodistas. p.1. 
diendo a lo público, sino también cotidiana y de acceso a todos, para que no quede relegada a especialistas o diplomados, sino, que todo agente sanitario, como primer responsables de hacer respetar y cumplir los derechos de los pacientes, y de implementar el consentimiento informado; sean ellos, los verdaderos bioeticistas públicos, y sientan que la bioética es parte esencial y estructurante en su ser y quehacer como agente en salud y generando la excelencia de su profesión.

\section{REFERENCIAS BIBLIOGRÁFICAS}

- ASTARITA, Martín, BONIFACINO, Santiago y DEL COGLIANO, Natalia (2012): “Relaciones intergubernamentales (RIGS) en la administración pública Argentina", en ABAL MEDINA, Juan Manuel y CAO, Horacio: Manual de la nueva administración pública argentina. Editorial Ariel, Buenos Aires.

- CORTINA, Adela (1997), La ética de la sociedad civil. Grupo Anaya. S.A. Madrid

- CORTINA, Adela (2003), Ciudadanos del mundo. Alianza Editorial. Madrid.

- GAFO, Javier (2003), Bioética Teológica. Comillas Universidad. Madrid.

- GRACIA, Diego (2004), Como Arquero al Blanco". Editorial Tríscatela. Madrid

- RAWLS, John (1979), Teoría de la justicia. Fondo Cultural Económica. México.

- ROSS, David. (1994). Lo correcto y lo Bueno. Ediciones Sígueme. Salamanca.

- SIMON LORDA, Pablo (2000), El consentimiento informado, Editorial Tríscatela. Madrid.

- TEALDI, Juan Carlos (2007) Retos para la bioética en el camino de la salud púbica en América Latina en AGUDELO, Saúl (editor) Bioética y Salud Pública: Encuentros y Tensiones. National Graphics LTDA. Colombia.

\section{Documentos de fuentes electrónicas:}

- Organización de Naciones Unidas (ONU) 1948. Declaración Universal de Derechos Humanos. Tomado de https://www.un.org/es/universal-declaration-human-rights/. Fecha de consulta: 20.09.2019.

- Organización de las Naciones Unidas para la educación, la ciencia y la cultura. (UNESCO). 2016. Manual de Bioética para Periodistas. Tomado de https://unesdoc.unesco.org/ ark:/48223/pf0000243107 .Fecha de consulta: 20.09.2019

- Universidad Católica Argentina (UCA) . Barometro de la deuda social Argentina.(2016) La salud en población urbana argentina desde una mirada multidimensional de la pobreza. Tomado de http://wadmin.uca.edu.ar/public/ckeditor/2018-OBSERVATORIO-DOCUMENTO-INVESTIGACION-SALUD.pdf. Fecha de consulta: 20.09.2019 